\title{
Considerações na Anestesia de Animais de Laboratório
}

\author{
Guilherme Hammarstrom Dobler ${ }^{1}$, Márcio Gonçalves Ferreira ${ }^{2}$ \\ Gustavo Henrique Mendes Bedendo ${ }^{3}$, Fernando Silvério Ferreira da $\mathrm{Cruz}^{4}$
}

\begin{abstract}
RESUMO
A utilização de animais em pesquisas requer um pensamento crítico, julgamento e análise, principalmente pelo fato de que a dor e o estresse não são facilmente avaliados nos animais, no entanto é importante considerar que sentem dor tal qual os seres humanos. Objetivo: Fazer uma revisão sobre a anestesia em animais de laboratório, apontando as considerações com base em suas particularidades. A anestesia em animais de laboratório tem como diretriz conduzir os experimentos científicos com a mais rigorosa maestria, de modo a gerar mínimo estresse aos animais e, por outro lado, ter a possibilidade de coletar dados com excelência. Métodos: Esta revisão surge a partir de pesquisa nos bancos de dados Pubmed, SciELO e Bireme, de consulta literária. Os achados apontam para as principais particularidades entre as espécies e técnicas de anestesiologia utilizadas. Resultados e Discussão: Foram descritos os principais fármacos e vias para utilização em camundongos, ratos Wistar e coelhos. Os resultados encontrados nesta revisão confirmam a necessidade de compreender as diferenças entre as espécies e o conhecimento farmacológico para realizar a conduta anestésica adequada para cada espécie.
\end{abstract}

Palavras-chave: Analgesia. Anestesia. Ratos. Camundongos.

\section{CONSIDERATIONS IN LABORATORY ANIMALS ANESTHESIA}

\section{ABSTRACT}

The use of animals in research requires critical thinking, judgment and analysis, mainly because pain and stress are not easily evaluated in animals. However it is important to consider that they feel pain equal to humans. Objective: Review about laboratory animal anesthesia making appointments based in their specificity. Anesthesia in laboratory animals has as guideline to conduct the scientific experiments with the most rigorous mastery, in order to generate minimum stress to the animals and, on the other hand, to be able to collect data with excellence. Methods: This review arises from research in databases as Pubmed, SciELO and Bireme, and from literary review. Results and Discussion: The findings point to the main peculiarities among the species and techniques of anesthesiology used in the present. The main drugs and routes for use in mice, Wistar rats and rabbits have been described. The results found in this review confirm the need to understand the differences between the species and the pharmacological knowledge to perform the appropriate anesthetic management for each species. Keywords: Analgesia. Anesthesia. Rats. Mices.

Recebido em: 14/3/2018

Alterações requeridas em: $25 / 10 / 2018$

Aceito em: 10/3/2019

\footnotetext{
${ }^{1}$ Graduando em Medicina Veterinária. Universidade Regional do Noroeste do Estado do Rio Grande do Sul - Unijuí. ghammars@asu.edu

2 Graduando em Medicina Veterinária. Universidade Regional do Noroeste do Estado do Rio Grande do Sul - Unijuí. marcio.unijui@gmail.com

${ }^{3}$ Graduando em Medicina Veterinária. Universidade Regional do Noroeste do Estado do Rio Grande do Sul - Unijuí. ghmbedendo@gmail.com

${ }^{4}$ Professor Doutor do Curso de Medicina Veterinária. Departamento de Estudos Agrários da Universidade Regional do Noroeste do Estado do Rio Grande do Sul - Unijuí. fernandosfcruz@hotmail.com
} 


\section{INTRODUÇÃO}

Coelhos e roedores são animais muito utilizados em pesquisa, e são frequentemente encaminhados a médicos veterinários para avaliação e tratamento. A anestesia em pets exóticos é frequentemente necessária para procedimentos diagnósticos e cirúrgicos, e está associada com um maior risco pré-operatório em coelhos e roedores, quando comparados com cães e gatos (WENGER, 2012).

Vários caminhos foram tomados para aperfeiçoamento da anestesiologia em animais de laboratório. Segundo Pompeu (2009), no início do século 21 ocorreu um grande acúmulo de conhecimentos básicos e de critérios das características neuroanatômicas e neurofisiológicas dos animais, e também uma compreensão da fisiologia da dor. Tais informações apoiam o ponto de vista segundo o qual os animais registram, transportam, processam e modulam os sinais nociceptivos de forma semelhante à espécie humana.

A utilização de animais em pesquisas requer pensamento crítico, julgamento e análise (COUNCIL, 2010), principalmente pelo fato de que a dor e o estresse não são facilmente avaliados nos animais, no entanto é importante considerar que sentem dor tal qual os seres humanos. Nesse sentido, muitos princípios têm sido propostos para nortear o emprego de organismos vertebrados em experimentos científicos (GOLDBERG, 2010).

Devido às diferenças fisiológicas entre os animais de laboratório é preciso enfatizar que os padrões para monitoração e cuidados com estes precisam ser extremamente acurados, pois problemas como hipotermia podem prolongar a recuperação do animal, provocando alterações fisiológicas e assim causar impactos nos objetivos da pesquisa (FLECKNELL et al., 2014). A monitoração deve ser constante, o uso de equipamentos específicos é de grande valor, no entanto algumas espécies ainda possuem limitações quanto à realização de procedimentos específicos relacionados à espécie (GOLDBERG, 2010).

De forma geral, em coelhos o uso de equipamentos de monitoração é simples e pode ser extrapolado de outras espécies; já com animais menores é o oposto. Entre os fatores que podem ser citados em relação à dificuldade de monitoração em animais de pequeno porte estão a alta frequência cardíaca, que algumas vezes pode acabar excedendo os parâmetros de alguns monitores; sua baixa força de contração também pode ser um problema, muitas vezes não sendo detectada, e principalmente seu corpo limita- do, que por ser pequeno alguns procedimentos, como monitoração invasiva da pressão arterial, não pode ser realizada (FLECKNELL et al., 2014).

Alguns dos fatores que aumentam a mortalidade dos roedores e coelhos, quando comparados com animais de companhia, são o estresse na indução da anestesia, o risco de hipotermia, a qual está associada a maior superfície corpórea para relação de volume, infecções respiratórias subclínicas em coelhos por Pausterella, doenças pré-operatórias envolvendo o trato digestório, respiratório e distúrbios hidroeletrolíticos, número menor de veias de fácil acesso para cateterização, além de a intubação endotraqueal ser mais difícil tecnicamente (WENGER, 2012).

Atualmente existe uma diversificada gama de fármacos que possibilitam anestesia e analgesia para os diferentes animais utilizados em laboratório (BRASIL, 2013). Salienta-se que a ciência pós-moderna primordialmente visa a utilizar técnicas/modelos experimentais que minimizem o uso de animais vertebrados, e, quando impreterivelmente necessários, estes sejam manuseados com ética, procedimentos adequados e indolores (GOLDBERG, 2010). No sentido de verificar algumas particularidades e as principais técnicas anestésicas utilizadas em ratos, camundongos e coelhos, este trabalho foi desenvolvido.

\section{METODOLOGIA}

O presente estudo consiste em uma revisão da literatura, realizada por meio de uma pesquisa nos bancos de dados Pubmed, SciELO e Bireme, e de consulta literária. Foram selecionados somente artigos completos, diretrizes e manuais para anestesia de animais, nos principais idiomas de publicações científicas das últimas duas décadas, em que constasse anestesia de animais em laboratório nas palavras-chave, título ou resumo. Os animais escolhidos para integrar esta revisão foram ratos Wistar, camundongos e coelhos, observando-se que a escolha ocorreu por serem os animais vertebrados mais representativos nas pesquisas com animais de laboratório.

Para a categorização dos estudos utilizou-se a técnica de análise de conteúdo, incluindo etapas como: pré-análise, exploração do material e tratamento dos resultados (BARDIN; RETO; PINHEIRO, 1979). Dessa forma, os principais aspectos de cada artigo selecionado foram colocados em uma tabela, para a realização de uma análise crítica dos parâmetros avaliados e dos resultados neles contidos. 


\section{RESULTADOS E DISCUSSÃO}

Primeiramente é importante ressaltar que não existe um agente anestésico ideal ou uma técnica perfeita para ser posta em prática, porém existem os mais apropriados para as diferentes situações. Quando selecionado um agente anestésico e método de administração, procura-se alcançar objetivos como minimizar o estresse sofrido pelo animal e a obtenção de resultados experimentais confiáveis (COUNCIL, 2010).

A associação de cetamina e xilazina foi muito popular no passado, mas a propriedade analgésica dessa associação é insuficiente para cirurgias maiores e em muitos casos pode desenvolver severa hipotensão, contribuindo para a alta mortalidade (WYATT; SCOTT; RICHARDSON 1989; POPILSKIS et al., 1991; DUPRAS et al., 2001; HENKE et al., 2005). Gonzáles et al. (2002) relataram o aumento das concentrações plasmáticas de Alanina Aminotransferase (ALT) e Aspartato Aminotransferase (AST), imputando à hipotensão causada pela xilazina, pois a cetamina isolada não altera o fluxo hepático e é incapaz de reverter a hipotensão causada pela xilazina. As concentrações de creatinina e ureia nitrogenada sanguínea após a administração de cetamina e xilazina também se elevaram, resultado da diminuição do fluxo sanguíneo renal e consequente diminuição da filtração glomerular, devido às alterações na pressão arterial.

A associação de tiletamina-zolazepam também é muito utilizada para anestesia em coelhos. Essa combinação associada à xilazina também mostrou depressão cardiovascular e respiratória (POPILSKIS et al., 1991). Existem relatos de que a tiletamina seja nefrotóxica em coelhos, podendo causar necrose tubular e nefrose nas doses comumente utilizadas para a indução anestésica ou contenção química (BRAMMER et al., 1991; DOERNING et al., 1992).

Nesse sentido, os procedimentos invasivos em animais devem ser realizados com agentes anestésicos adequados, seja a anestesia local ou geral (BRASIL, 2013), devendo-se ter cautela quanto à escolha e administração dos agentes anestésicos, tranquilizantes ou analgésicos de modo a utilizar-se de compostos apropriados para a espécie (SPINOSA; GÓRNIAK; BERNARDI, 2011), devendo-se conhecer a farmacologia dos anestésicos, as particularidades anatomofisiológicas das espécies, o objetivo e duração do procedimento. A monitoração durante a cirurgia e os cuidados pós-operatórios também são importantes para a condução do experimento e a qualidade de vida do animal (COUNCIL, 2010; BRASIL, 2013), sendo importante o conhecimento dos valores fisiológicos dos principais parâmetros, a fim de identificar as alterações durante e após a anestesia (Tabela 1). Assim, consegue-se determinar a necessidade de analgesia e qual a interferência nos sistemas.

\section{Particularidades}

\section{Ratos Wistar}

$\mathrm{O}$ rato Wistar possui corpo fusiforme e uma cauda que em muitas raças/linhagens pode chegar a medir mais em comprimento do que o corpo (CLAU$\mathrm{SE}$, 1993). Como outros roedores, não possuem glândulas sudoríparas; desse modo, em ambientes quentes procuram locais com sombras ou cavam tocas (ambientes mais frios), o que pode interferir com a temperatura corpórea durante o procedimento anestésico, provocando hipotermia. Os ratos são excelentes para o desenvolvimento de pesquisas, pois são animais uniformes, que adaptam-se facilmente aos ambientes, reproduzem-se rapidamente, são facilmente manuseados e os resultados podem ser reproduzidos em todo território mundial (CLAUSE, 1993).

De acordo com Flecknell et al. (2014), a medicação pré-anestésica (MPA) muitas vezes não é utilizada em roedores, uma vez que a anestesia geralmente é induzida por uma câmara anestésica, ou administrada por via intraperitoneal (IP) devido à relativa rapidez e dificuldade em punção venosa ou subcutânea (SC). Em casos, contudo, em que será administrado o isofluorano, é recomendado seu uso, pois o fármaco

Tabela 1 - Principais dados de espécies de vertebrados mais utilizados atualmente em laboratórios

\begin{tabular}{lccc}
\hline & Camundongo & Rato & Coelho \\
\hline Peso adulto $(\mathrm{g})$ & $25-40$ & $200-400$ & $2000-4000$ \\
Temperatura corpórea $\left({ }^{\circ} \mathrm{C}\right)$ & $36,5-38$ & $36-37,5$ & $38-39,5$ \\
Frequência respiratória $(\mathrm{mpm})$ & $80-200$ & $70-115$ & $30-60$ \\
Frequência cardíaca $(\mathrm{bpm})$ & $350-750$ & $250-450$ & $135-325$ \\
Pressão arterial $(\mathrm{mmHg})$ & $80-100$ & $90-110$ & $60-90$ \\
\hline
\end{tabular}

Fonte: Adaptado de FLECKNELL et al., 2014. 
parece ser irritante para a espécie. Ademais, a manipulação excessiva pode ser um fator desencadeante de estresse.

A maior diferença observada no uso de anestésicos injetáveis em pequenos roedores é a dificuldade do acesso venoso, o que faz com que a anestesia e analgesia sejam fornecidas por outras vias. Flecknell et al. (2014) destacam que quando necessárias, são administradas em doses únicas por via IP, SC ou intramuscular (IM). Devido ao seu pequeno porte e alta taxa metabólica, doses elevadas de certos medicamentos, como cetamina, são necessários para atingir um estado de inconsciência, porém gera um volume muito elevado e caso administrado por via IM pode causar lesões. Tendo isso em mente, deve-se avaliar qual associação anestésica é mais viável para cada situação.

\section{Camundongos}

É um animal suscetível a alterações nas condições ambientais, pequenas variações na temperatura do ambiente e as respostas fisiológicas do animal podem acabar alteradas, correndo grande risco de entrarem em um estado de desidratação, visto que são mais sensíveis em relação a outras espécies (KO; LUCA, 2009).

Os protocolos de MPA para camundongos e ratos são similares, mudando apenas as doses dos fármacos empregados (SPINOSA; GÓRNIAK; BERNARDI, 2011). As características citadas anteriormente para ratos aplicam-se também para camundongos (CLAUSE, 1993). Na maioria dos casos, a MPA pode ser excluída e ser realizada contenção química à base de anestésicos dissociativos, como cetamina e tiletamina, uma vez que a contenção física repetidas vezes e as múltiplas injeções podem aumentar o grau de estresse e o risco de lesões.

Os camundongos também passam pelos mesmos problemas que ratos com o emprego de anestésicos injetáveis. A cetamina, como foi citado anteriormente, sozinha não é utilizada, porém ao se associar com outros fármacos, planos anestésicos podem ser obtidos. Ao se associar com tranquilizantes, produzem anestesia leve, já quando associada com agonistas $\alpha-2$ adrenérgicos, podem produzir planos anestésicos cirúrgicos, mas produzem depressão cardiorrespiratória intensa (FLECKNELL et al., 2014), podendo ser empregados benzodiazepínicos para reduzir a depressão. Os fármacos e combinações, e as respectivas doses recomendadas para as espécies supracitadas, estão listados na Tabela 2.
Tabela 2 - Principais fármacos e doses $(\mathrm{mg} / \mathrm{kg}$ ) utilizadas em protocolos anestésicos em camundongos e ratos

\begin{tabular}{|c|c|c|c|}
\hline Fármacos & Vias & Camundongos & Ratos \\
\hline $\begin{array}{c}\text { MPA } \\
\text { Atropina } \\
\end{array}$ & IP, SC & $0,02-0,05$ & $0,05-0,1$ \\
\hline $\begin{array}{c}\text { Diazepam } \\
\text { Midazolam }\end{array}$ & IP & 5 & $2-4$ \\
\hline Acepromazina & IP, SC & $2-5$ & $1-2$ \\
\hline Xilazina & $\mathrm{IP}, \mathrm{SC}$ & $5-10$ & $1-5$ \\
\hline $\begin{array}{l}\text { Medetomidina } \\
\text { Cetamina }\end{array}$ & $\begin{array}{l}\text { IP, SC } \\
\text { IP, SC }\end{array}$ & $\begin{array}{c}0,1-0,3 \\
22-44\end{array}$ & $\begin{array}{c}0,1-0,3 \\
22-50\end{array}$ \\
\hline \multicolumn{4}{|l|}{ Anestesia } \\
\hline Tiopental & $\mathrm{IP}$ & $40-60$ & $20-30$ \\
\hline $\begin{array}{l}\text { Tiletamina } \\
\text { Zolazepam }\end{array}$ & IP & $80-100$ & $20-40$ \\
\hline $\begin{array}{c}\text { Cetamina } \\
\text { Xilazina }\end{array}$ & IP & $\begin{array}{c}100-200 \\
5-15\end{array}$ & $\begin{array}{c}75-100 \\
10\end{array}$ \\
\hline $\begin{array}{l}\text { Cetamina } \\
\text { Diazepam }\end{array}$ & IP & $\begin{array}{c}200 \\
5\end{array}$ & $\begin{array}{c}40-80 \\
5-10\end{array}$ \\
\hline $\begin{array}{c}\text { Cetamina } \\
\text { Medetomidina } \\
\text { Halotano } \\
\text { Isofluorano }\end{array}$ & $\begin{array}{c}\text { IP } \\
\text { Inalat. } \\
\text { Inalat. }\end{array}$ & $\begin{array}{c}75 \\
1 \\
1,3 \mathrm{~V} \% \\
2 \mathrm{~V} \%\end{array}$ & $\begin{array}{c}75 \\
0,5 \\
1,3 \mathrm{~V} \% \\
2 \mathrm{~V} \%\end{array}$ \\
\hline \multicolumn{4}{|l|}{ Analgésicos } \\
\hline Butorfanol & $\mathrm{IP}, \mathrm{SC}$ & $1-5$ & $0,5-2$ \\
\hline Metadona & SC & - & $0,5-3$ \\
\hline Morfina & $\mathrm{IP}, \mathrm{SC}$ & $5-10$ & $1,5-6$ \\
\hline Meperidina & $\mathrm{IP}, \mathrm{SC}$ & $20-60$ & $10-20$ \\
\hline Codeína & $\mathrm{SC}, \mathrm{PO}$ & 50 & 20 \\
\hline Meloxicam & $\mathrm{SC}, \mathrm{PO}$ & 5 & 1 \\
\hline $\begin{array}{l}\text { Flunixim meglu- } \\
\text { mine }\end{array}$ & $\mathrm{SC}$ & $2-3$ & $1-2$ \\
\hline $\begin{array}{l}\text { Cetoprofeno } \\
\text { Carprofeno } \\
\text { Ibuprofeno }\end{array}$ & $\begin{array}{l}\mathrm{SC}, \mathrm{PO} \\
\mathrm{SC} \\
\mathrm{PO}\end{array}$ & $\begin{array}{c}5 \\
5 \\
40\end{array}$ & $\begin{array}{c}5 \\
5 \\
30\end{array}$ \\
\hline
\end{tabular}

Fonte: Elaborada pelos autores.

\section{Coelhos}

Particularmente, os coelhos são animais de risco por serem considerados sensíveis aos anestésicos (HENKE et al., 2005). Segundo Pompeu (2009), coelhos são animais difíceis e imprevisíveis de serem anestesiados pelos seguintes motivos: seu centro respiratório é sensível e o risco de apneia é maior na espécie, as doses anestésicas e letais são muito próximas e há variabilidade entre coelhos em relação aos anestésicos, mesmo dentro da mesma raça. Outro agravante na anestesia de coelhos é que eles podem desenvolver distúrbios gastrintestinais durante o pós-operatório se forem mantidos em jejum e realizam a coprofagia. Como a espécie possui alto metabolismo, assim como ratos e camundongos, não existe a neces- 
sidade de se realizar jejum, mas deve-se atentar para a possibilidade de aspiração, uma vez que algumas medicações provocam o relaxamento da cárdia.

Durante a anestesia é necessário o monitoramento do animal, pois padrões como frequência e amplitude respiratória, frequência cardíaca, pressão arterial, coloração de mucosas, reflexos protetores, indicam o plano anestésico em que se encontra (POMPEU, 2009).

Ao contrário das outras espécies, a MPA em coelhos é de extrema importância, uma vez que são animais que ficam estressados com facilidade, pelo manuseio e contenção, e o próprio procedimento de indução anestésica pode ser considerado estressante. É recomendado que o fármaco seja administrado antes da remoção do animal do seu ambiente. Os protocolos recomendados estão listados na Tabela 3.

Tabela 3 - Principais fármacos e doses $(\mathrm{mg} / \mathrm{kg}$ ) utilizadas em protocolos anestésicos em coelhos

\begin{tabular}{|c|c|c|}
\hline Fármacos & Vias & Coelhos \\
\hline \multicolumn{3}{|l|}{ MPA } \\
\hline Atropina & SC, IM & $1-2$ \\
\hline $\begin{array}{l}\text { Diazepam } \\
\text { Midazolam }\end{array}$ & IP, IM & $4-10$ \\
\hline Acepromazina & IP, SC & $1-2$ \\
\hline Xilazina & IP, SC & $3-5$ \\
\hline Medetomidina & IP, SC & $0,1-0,3$ \\
\hline Cetamina & $\mathrm{IM}$ & $15-20$ \\
\hline \multicolumn{3}{|l|}{ Anestesia } \\
\hline Tiopental & IV & $20-28$ \\
\hline $\begin{array}{l}\text { Tiletamina } \\
\text { Zolazepam }\end{array}$ & IM & $\begin{array}{c}\text { Não recomen- } \\
\text { dado }\end{array}$ \\
\hline $\begin{array}{c}\text { Cetamina } \\
\text { Xilazina }\end{array}$ & IM & $\begin{array}{c}35-50 \\
5-10 \\
\end{array}$ \\
\hline $\begin{array}{l}\text { Cetamina } \\
\text { Diazepam }\end{array}$ & IM & $\begin{array}{c}20-40 \\
2-5\end{array}$ \\
\hline $\begin{array}{c}\text { Cetamina } \\
\text { Medetomidina } \\
\end{array}$ & IM & $\begin{array}{l}25 \\
0,5 \\
\end{array}$ \\
\hline Propofol & IV & $7,5-15$ \\
\hline Halotano & Inalat. & $1,5 \mathrm{~V} \%$ \\
\hline Isofluorano & Inalat. & $3 \mathrm{~V} \%$ \\
\hline \multicolumn{3}{|l|}{ Analgésicos } \\
\hline Butorfanol & IV, IM, SC & $0,1-0,5$ \\
\hline Morfina & IV, IM, SC & $2-5$ \\
\hline Meperidina & IV, IM, SC & $10-20$ \\
\hline Carprofeno & $\mathrm{PO}$ & $1,5-3$ \\
\hline Flunixim meglumine & SC & $1-1,5$ \\
\hline Piroxicam & $\mathrm{PO}$ & $0,2-0,3$ \\
\hline Meloxicam & SC & $0,6-1$ \\
\hline
\end{tabular}

Fonte: Elaborada pelos autores.

\section{Agentes Anestésicos Inalatórios}

A escolha de fármacos inalatórios utilizados durante os procedimentos irá determinar a qualidade da indução anestésica, do período transoperatório e da recuperação no pós-operatório. Esse tipo de anestesia proporciona um maior controle do plano anestésico, um rápido retorno da anestesia quando comparada com outros procedimentos, pois possui menor metabolização e elimina o agente mais rapidamente pelas vias aéreas (POMPEU, 2009).

Em roedores a administração de agentes inalatórios é fácil, uma vez que são pequenos, basta uma câmara de indução anestésica para anestesiar o animal, podendo-se também utilizar máscara facial. Em relação à anatomia da orofaringe do coelho, destacam-se particularidades como estreita cavidade oral, dentes incisivos avantajados, língua grossa e protusa e pouca mobilidade da articulação temporomandibular, que tornam difícil a intubação nessa espécie (SMITH et al., 2004; BATEMAN et al., 2005). Essa manobra pode causar trauma de laringe, laringoespasmo e lesão traqueal (BATEMAN et al., 2005), exigindo experiência e destreza do anestesista.

Uma alternativa à intubação endotraqueal é a utilização da máscara facial, o que não previne a obstrução das vias aéreas (BATEMAN et al., 2005), podendo ainda ser muito estressante para o paciente. Outra opção é o emprego de máscara laríngea, a qual se mostra mais adequada, uma vez que se adapta à laringe evitando lesão, laringoespasmo e obstrução das vias aéreas.

\section{Principais Agentes Anestésicos Inalatórios}

Éter etílico: Anestésico fora de uso, uma vez que sua administração não promovia consistência em termos de concentração inalada e causava irritação e estresse ao animal. Era administrado com o anestésico líquido embebido em algodão colocado dentro de uma câmara anestésica (FLECKNELL et al., 2014).

Halotano: Pode ser utilizado e é eficaz para a maioria dos animais de laboratório, uma vez que seus efeitos são similares ao observado em animais de companhia e produção. Seu uso é mais voltado em estudos neurofisiológicos, dado que seus efeitos no sistema nervoso são menos pronunciados (FLECKNELL et al., 2014). Encontra-se fora de uso atualmente, por questão de custo e alterações metabólicas importantes que podem causar adulterações no estudo.

Isofluorano: Considerado o fármaco mais utilizado dentre os agentes inalatórios em animais de laboratório, visto sua menor interferência em estudos 
sobre farmacocinética e farmacodinâmica. Sua rápida indução e recuperação fazem com que seja fácil o controle da profundidade anestésica, seja para procedimentos curtos ou em longo prazo (FLECKNELL et al., 2014).

Desfluorano: Pouco utilizado entre as espécies de laboratório, seus efeitos são similares aos observados em animais de companhia e pode ser usado para promoção de anestesia estável com indução e recuperação rápida. Apresenta boa tolerância para indução anestésica em coelhos (FLECKNELL et al., 2014). Ademais, necessita de um sistema específico de vaporização para o agente, o que o torna muito dispendioso.

Sevofluorano: Pouco difundido, porém suas características como velocidade e qualidade de indução são boas para uma variedade de estudos, porém para manter o plano anestésico cirúrgico podem ser necessárias altas concentrações do fármaco e isso está associado a uma alta mortalidade (FLECKNELL et al., 2014).

Óxido nitroso: Quando sozinho não produz anestesia, posto que sua concentração alveolar mínima (CAM) é de $100 \%$, logo, é empregado como parte do protocolo de uma anestesia balanceada. Seu ponto negativo é o fato de não ser absorvido pelo sistema de filtro de gases por carvão ativado. Já pelo lado positivo são situações muito específicas, como uma anestesia prolongada para estudos neurofisiológicos (FLECKNELL et al., 2014).

\section{CONSIDERAÇÕES FINAIS}

Os resultados encontrados confirmam a necessidade de compreender as diferenças entre as espécies para realizar a conduta anestésica adequada a cada uma, apesar das semelhanças entre si. Desta forma, com a clara identificação das particularidades e a sua compreensão, poderão ser elaborados protocolos adequados a cada espécie, permitindo uma anestesia mais segura, a analgesia adequada e com menor interferência na pesquisa.

\section{REFERÊNCIAS}

BARDIN, L.; RETO, L. A.; PINHEIRO, A. Análise de conteúdo. Lisboa: Edição 70, 1979.

BATEMAN, L. et al. Comparison between facemask and laryngeal mask airway in rabbits during isoflurane anesthesia. Veterinary Anaesthesia and Analgesia, v. 32, p. 280-288, 2005.

BRAMMER, D. W. et al. Anesthetic and nephrotoxic effects of telazol in New Zealand white rabbits. Laboratory Animal Science, v. 41, p. 432-435, 1991.
BRASIL. Ministério da Ciência, Tecnologia e Inovação. Diretriz brasileira para o cuidado e a utilização de animais para fins científicos e didáticos: DBCA. Brasília: Concea, 2013. p. 1-50.

BRONAUGH, R. L.; STEWART, R. F.; CONGDON, E. R. Methods for in vitro percutaneous absorption studies II. Animal models for human skin. Toxicology and Applied Pharmacology, v. 62, n. 3, p. 481-488, 1982.

CARDOSO, T. A. O. Biossegurança no manejo de animais em experimentação. In: ODA, L.; ÁVILA, S. M. Biossegurança em laboratórios de saúde pública. Brasília: Fiocruz, 1998. p. 11-14.

CLAUSE, B. T. The Wistar rat as a right choice: Establishing mammalian standards and the ideal of a standardized mammal. Journal of the History of Biology, v. 26, n. 2, p. 329-349, 1993.

COUNCIL, N. R. Guide for the Care and Use of Laboratory Animals. Eighth Edition. Washington, D. C, 2010.

DOERNING, B. J. et al. Nephrotoxicity of tiletamine in New Zealand white rabbits. Laboratory Animal Science, 42, p. 267-269, 1992.

DUPRAS, J. et al. Anesthesia of the New Zealand rabbit using the combination of tiletamine-zolazepam and ketamine-midazolam with or without xylazine. The Canadian Veterinary Journal, v.42, p. 455-460, 2001.

FLECKNELL, P A. et al. Animais de Laboratório. In: TRANQUILLI, William J.; THURMON, John C.; GRIMM, Kurt A. (org.). Lumb \& Jones: Anestesiologia e Analgesia Veterinária. 4. ed. São Paulo: Roca, 2014. p. 837-859.

GOLDBERG, A. M. The Principles of Humane Experimental Technique: Is It Relevant Today? Altex, p. 25-27, 2010.

GONZALES, G. A. et al. Changes in hepatic and renal enzyme concentrations and heart and respiratory rates in New Zealand white rabbits after anesthetic treatments. Contemporary Topics in Laboratory Animal Science, v. 41, p. 30-32, 2002.

HENKE, J. et al. Comparative study of three intramuscular anaesthetic combinations (medetomidine/ketamine, medetomidine/fentanyl/midazolam and xylazine/ketamine) in rabbits. Veterinary Anaesthesia and Analgesia, v. 32, p. 261270, 2005.

KO, Gui Mi; LUCA, Rosália Regina de. Camundongo. In: LAPCHIK, Valderez Bastos Valero.; MATTARAIA, Vania Gomes de Moura.; KO, Gui Mi; Cuidados e manejo de animais de laboratório. São Paulo: Ateneu, 2009. p. 561-574.

LIGGITT, D. Comparative anatomy and histology: a mouse and human atlas. First ed. Seattle, WA, USA: Elsevier Inc. All, 2012.

LOVEDAY, D. E. An in vitro method for studying percutaneous absorption. Journal of the Society of Cosmetic Chemists, p. 224-239, 1961.

POMPEU, E. Analgesia e anestesia. In: LAPCHIK, Valderez Bastos Valero; MATTARAIA, Vania Gomes de Moura; KO, Gui Mi. Cuidados e manejo de animais de laboratório. Ateneu: São Paulo, 2009. p. 561-574. 
POPILSKIS, S. J. et al. Comparison of xylazine with tiletamine-zolazepam (telazol) and zylazine-ketamine anesthesia in rabbits. Laboratory Animal Science, 41, p. 51-53, 1991.

SMITH, J. C. et al. Endotracheal tubes versus laryngeal mask airways in rabbit inhalation anesthesia: ease of use and waste gas emissions. Contemporary Topics in Laboratory Animal Science, v.43, n. 4, p. 22-25, 2004.

SPINOSA, H. DE S.; GÓRNIAK, S. L.; BERNARDI., M. M. Farmacologia aplicada à medicina veterinária. 5 . ed. Rio de Janeiro: Editora Guanabara Koogan, 2011.
URBAN, B. W.; FRIEDERICH, P. Anesthetic mechanisms in-vitro and in general anesthesia. Toxicology Letters, v. 100-101, p. 9-16, 1998.

WENGER, S. Anesthesia and analgesia in rabitts and rodents. Journal of Exotic Pet Medicine, v. 21 (1), p. 7-16, 2012.

WYATT, J. D.; SCOTT, R. A.; RICHARDSON, M. E. The effects of prolonged ketamine-xylazine intravenous infusion on arterial blood $\mathrm{pH}$, blood gases, mean arterial blood pressure, heart and respiratory rates, rectal temperature and reflex in the rabbit. Laboratory Animal Science, 39, p. 411-416, 1989. 